The true structural periodicities and superspace group descriptions of the prototypical incommensurate composite materials: Alkane/urea inclusion compounds

This content has been downloaded from IOPscience. Please scroll down to see the full text. 2016 EPL 11656001

(http://iopscience.iop.org/0295-5075/116/5/56001)

View the table of contents for this issue, or go to the journal homepage for more

Download details:

IP Address: 131.251.254.109

This content was downloaded on 07/02/2017 at 10:44

Please note that terms and conditions apply.

You may also be interested in:

Nonadecane/urea inclusion compound structure

P Rabiller, J Etrillard, L Toupet et al.

P-T phase diagram of nonadecane-urea inclusion compound

B. Toudic, P. Rabiller, L. Bourgeois et al.

Low-frequency dynamics in molecular incommensurate composite: Specific heat of nonadecane/urea inclusion compound

J. Etrillard, J. C. Lasjaunias, B. Toudic et al.

Dynamics of alkyl-type chains in crystals

F Guillaume, A El Baghdadi and A J Dianoux

Neutron scattering investigations of guest molecular dynamics in alpha , omega -dibromoalkane-urea inclusion compounds

F Guillaume, S P Smart, K D M Harris et al.

Structure of the charge-density wave in(TaSe4)2I

Sander van Smaalen, Erwin J Lam and Jens Lüdecke

$(3+1) D$ superspace description of the incommensurate modulation in the premartensite phase of Ni2MnGa: a high resolution synchrotron x-ray powder diffraction study

Sanjay Singh, J Nayak, Abhishek Rai et al. 


\title{
The true structural periodicities and superspace group descriptions of the prototypical incommensurate composite materials: Alkane/urea inclusion compounds
}

\author{
Michel Couzi ${ }^{1(a)}$, François Guillaume $^{1(\mathrm{~b})}$, Kenneth D. M. Harris $^{2(\mathrm{c})}$, Benjamin A. Palmer ${ }^{2,3}$, \\ Kirsten Christensen ${ }^{4,5}$ and Stephen P. Collins ${ }^{4}$ \\ 1 Université de Bordeaux, CNRS, ISM UMR 5255 - 351 cours de la Libération, F-33405 Talence Cedex, France \\ 2 School of Chemistry, Cardiff University - Park Place, Cardiff CF10 3AT, Wales, UK \\ 3 Department of Structural Biology, Weizmann Institute of Science - Rehovot 7610001, Israel \\ 4 Diamond Light Source, Harwell Science and Innovation Campus - Didcot, Oxfordshire OX11 ODE, England, UK \\ 5 Inorganic Chemistry Laboratory - South Parks Road, Oxford OX1 3QR, England, UK
}

received 27 July 2016; accepted in final form 9 December 2016

published online 13 January 2017

PACS 61.44.Fw - Incommensurate crystals

PACS $61.50 . \mathrm{Ks}$ - Crystallographic aspects of phase transformations; pressure effects

PACS 64.70.K-- Solid-solid transitions

\begin{abstract}
The prototypical family of incommensurate composite materials are the $n$-alkane/urea inclusion compounds, in which $n$-alkane guest molecules are arranged in a periodic manner along one-dimensional tunnels in a urea host structure, with an incommensurate relationship between the periodicities of the host and guest substructures along the tunnel. We develop interpretations of the structural periodicities, superspace group descriptions and symmetry properties of the lowtemperature phases of $n$-alkane/urea inclusion compounds, based in part on a high-resolution synchrotron single-crystal X-ray diffraction study of $n$-nonadecane/urea. Specifically, we prove that, on passing from phase I to phase II, the C-centering of the orthohexagonal unit cell is lost for both the host and guest substructures, and that the symmetries of all phases I, II and III are described completely by $(3+1)$-dimensional superspace groups.
\end{abstract}

Copyright (c) EPLA, 2016

Aperiodic crystals are defined as materials that do not have 3-dimensional translational periodicity, yet exhibit sharp Bragg maxima in their X-ray diffraction patterns. Among the various classes of aperiodic materials, incommensurate solids [1] and quasicrystals [2] have received particular attention both from the fundamental perspectives of diffraction physics and structural science and with regard to their potential applications $[3,4]$.

Host/guest composite materials are constructed from two interpenetrated substructures. In many cases, the host and guest subsystems share common crystallographic axes in three-dimensional space, and are commensurate structures. However, in some cases, there is an irrational relationship between the periodicities of the host and guest substructures, at least in one direction, and such materials are classified as incommensurate structures.

\footnotetext{
(a) E-mail: michel.couzi@u-bordeaux .fr

(b) E-mail: francois.guillaume@u-bordeaux.fr

(c) E-mail: HarrisKDM@cardiff .ac.uk
}

The prototypical family of incommensurate composite materials is the $n$-alkane/urea inclusion compounds [5-11], in which linear chain $n$-alkane guest molecules are arranged in a periodic manner along one-dimensional tunnels (diameter ca. $5.25 \AA$ ) that exist within the urea host structure, which is constructed from a helical hydrogenbonded arrangement of urea molecules (fig. 1(a)).

Urea inclusion compounds exhibit a wealth of interesting physico-chemical properties that are derived from their unique structural characteristics including crystal growth processes [12-16], X-ray dichroism [17] and X-ray birefringence [18]. A consequence of the incommensurateness of urea inclusion compounds is that the set of guest molecules may, in principle, slide along the host tunnel with essentially zero activation energy; this phenomenon has been harnessed in molecular transport experiments [19-22]. In order to fully understand the properties of these materials, it is imperative to establish an accurate description of their aperiodic structures. 


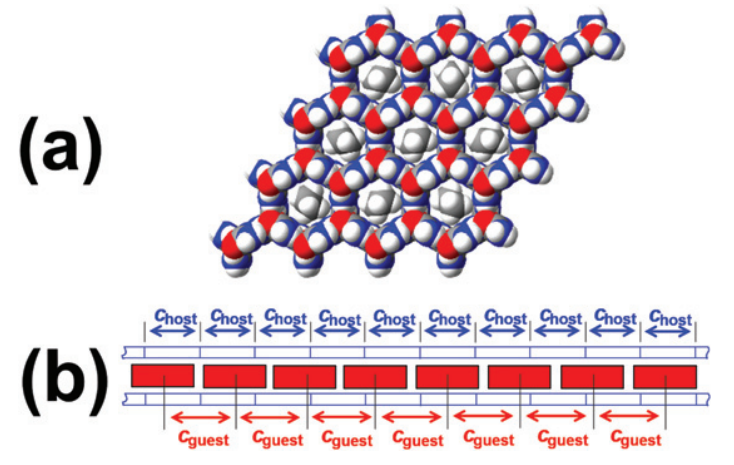

Fig. 1: (Colour online) (a) Structure of an $n$-alkane/urea inclusion compound at ambient temperature viewed along the tunnel axis. (b) Schematic representation of a tunnel inclusion compound viewed perpendicular to the tunnel axis, showing guest molecules (red) arranged along the host tunnel structure (blue). The periodic repeat distances of the host and guest substructures along the tunnel axis are denoted $\boldsymbol{c}_{\text {host }}$ and $\boldsymbol{c}_{\text {guest }}$, respectively.

The $n$-alkane/urea inclusion compounds are characterized by the following features at ambient temperature: i) a hexagonal urea tunnel structure $[5,6,8]$, ii) an incommensurate relationship $[6,8,11,23-43]$ between the periodicities of the host and guest substructures along the tunnel axis, and iii) dynamic disorder of the guest molecules [32,44-49] (reorientation about the tunnel axis and translations along the tunnel axis).

Focusing on the incommensurate properties of $n$ alkane/urea inclusion compounds, the misfit parameter $\gamma$ is defined as $\gamma=c_{\text {host }} / \boldsymbol{c}_{\text {guest }}$, where $\boldsymbol{c}_{\text {host }}$ and $\boldsymbol{c}_{\text {guest }}$ are the periodicities of the host and guest substructures along the tunnel direction, respectively (fig. 1(b)). Clearly, $\boldsymbol{c}_{\text {host }}$ depends on the pitch of the urea helix and $\boldsymbol{c}_{\text {guest }}$ depends on the length of the $n$-alkane guest molecule. For all $n$-alkane/urea inclusion compounds at ambient pressure, $c_{\text {host }} \approx 11.02 \AA[5,6]$. In this paper, we focus on the urea inclusion compounds containing $n$-nonadecane $\left[\mathrm{CH}_{3}\left(\mathrm{CH}_{2}\right)_{17} \mathrm{CH}_{3}\right]$ and $n$-hexadecane $\left[\mathrm{CH}_{3}\left(\mathrm{CH}_{2}\right)_{14} \mathrm{CH}_{3}\right]$ guest molecules.

At ambient temperature, the urea host substructure has space group $P 6_{1} 22$ (or $P 6_{5} 22$ ) [5,6]. The composite crystal achieves 6 -fold symmetry as a consequence of the $n$-alkane guests being distributed statistically in at least six equi-probable orientations. Under these conditions, the $n$-alkane substructure is described by space group $P 622$. This assignment is based on the fact $[7,29,50]$ that the $n$-alkane guest molecules exhibit 3 -dimensional ordering in which guest molecules in adjacent tunnels are located at the same set of positions ( $z$-coordinates) along the tunnel direction: i.e., the offset $\left(\Delta_{\mathrm{g}}\right)$, along the tunnel direction, between the positions of guest molecules in adjacent tunnels (defined in ref. [29]) is $\Delta_{\mathrm{g}}=0$.

It follows that the symmetry of the incommensurate composite material is described by the $(3+1)$-dimensional superspace group $P 6{ }_{1} 22(00 \gamma)$ [28-30] and four integer indices $(h, k, l, m)$ are required to index all Bragg peaks in the diffraction pattern:

$$
\boldsymbol{Q}_{\mathrm{hklm}}=h \boldsymbol{a}^{*}+k \boldsymbol{b}^{*}+l \boldsymbol{c}_{\mathrm{host}}^{*}+m \boldsymbol{c}_{\text {guest }}^{*} .
$$

The reflections $(h, k, l, m)$ can be subdivided into three classes according to whether $l$ and $m$ are zero or nonzero: i) $m=0$ : "main reflections" from the host substructure, which primarily contain information on the basic host structure, but also contain information on the incommensurate modulations within the guest substructure; ii) $l=0$ : "main reflections" from the guest substructure, which primarily contain information on the basic guest structure, but also contain information on the incommensurate modulations within the host substructure; iii) $l \neq 0$ and $m \neq 0$ : "satellite reflections" that arise due to the inter-modulation of the two substructures. Each $(h, k, 0,0)$ reflection is the superposition of a main reflection from each substructure, representing the common $\boldsymbol{a}^{*} \boldsymbol{b}^{*}$ reciprocal lattice plane for the host and guest substructures. The satellite reflections with $l \neq 0$ and $m \neq 0$ are typically very weak in comparison to the other types of reflection, and it is essential to use the high intensity of a synchrotron source in order to be able to observe and measure these satellite reflections in X-ray diffraction data.

Many techniques have been used to explore structural phase transitions in $n$-alkane/urea inclusion compounds $[8,25,28-30,32,50-57]$. Early studies reported that $n$-alkane/urea inclusion compounds undergo a single phase transition below ambient temperature [56] from the hexagonal phase I at ambient temperature to an orthorhombic phase II with space group $P 2_{1} 2_{1} 2_{1}$ at low temperature. It was later suggested [58] that this phase transition is governed by a simple pseudospin-phonon model due to an antiferro-ordering of the $n$-alkane guests affecting both the host shearing and guest orientation. A theoretical analysis [29] described the phase transitions within the framework of a $(3+1)$-dimensional superspace group description of all possible group-subgroup related host and guest sub-lattices. It followed that phase I is described by superspace group $P 6_{1} 22(00 \gamma)$ and phase II is described by superspace group $P 2_{1} 2_{1} 2_{1}(00 \gamma)$. In both phases I and II, the host and guest substructures have the same periodicities in the $\boldsymbol{a} \boldsymbol{b}$-plane, and the misfit parameter $\gamma$ along the $\boldsymbol{c}$-axis is the same. In the $\boldsymbol{a b}$-plane, the unit cell of phase II closely approximates the orthohexagonal description of the hexagonal unit cell of phase I (with orthorhombic lattice parameters $\boldsymbol{a}_{\circ}$ and $\boldsymbol{b}_{\mathrm{o}}$ related by: $\boldsymbol{a}_{\mathrm{\circ}} \approx \boldsymbol{b}_{\mathrm{o}} \sqrt{ } 3$ ), but with loss of C-centering.

A recent series of papers [11,36-43] has argued against this simple generalized description of the phase transitions in $n$-alkane/urea inclusion compounds. First, for $n$-nonadecane/urea $[11,36,38,42]$, in addition to the "classical" I $\leftrightarrow$ II phase transition (discussed above) at temperature $T_{1}$, another phase transition at a lower temperature $T_{2}$ was reported, corresponding to a weak thermal event in DSC data [59]. The new phase below $T_{2}$ is denoted 
phase III. Furthermore, it was reported that, for phase II, no "main reflections" (i.e., $(h, k, l, m)$ with $l=0$ and/or $m=0$ ) were observed for which $h+k$ is odd, leading to the conclusion that this phase is based on a C-centred orthorhombic unit cell. Surprisingly, however, several satellite reflections (i.e., $(h, k, l, m)$ with $l \neq 0$ and $m \neq 0$ ) were observed for values of $h$ and $k$ for which $h+k$ is odd $[36,38,42]$, implying that the C-centering is actually lost for the incommensurate inter-modulations. The positions of these satellite reflections along the $\boldsymbol{c}^{*}$-axis were rationalized by introducing an additional misfit parameter $\delta=0.090$ that coexists with the misfit parameter $\gamma=0.418$ at ambient pressure $[11,36,38,42]$ (note that the value $\gamma=0.428$ has been reported at the higher pressure of $0.5 \mathrm{GPa}[38]$ ), necessitating the description of the symmetry properties of phase II in terms of a $(3+2)$-dimensional superspace. In phase III, reflections for which $h+k$ is odd, attributed to the loss of C-centering of the orthorhombic unit cell, were observed both for main reflections and for satellite reflections.

These observations raise a seemingly impossible physical conundrum: how does the material undergo a phase transition (from phase I to phase II) involving a change in the inter-modulation of the two substructures (i.e., the loss of C-centering) that is not reflected by a corresponding change in the basic host structure or the basic guest structure (for which $\mathrm{C}$-centering is retained), particularly as it is the interactions between the two basic structures that ultimately cause the incommensurate inter-modulations?

The $(3+2)$-dimensional superspace groups proposed $[38,42]$ for phase II and phase III were $C 222_{1}(00 \gamma)$ $(10 \delta)$ and $P 2_{1} 2_{1} 2_{1}(00 \gamma)(00 \delta)$, respectively, with five independent indices $(h, k, l, m, n)$ required to index each reflection:

$$
\boldsymbol{Q}_{h k l m n}=h \boldsymbol{a}^{*}+k \boldsymbol{b}^{*}+l \boldsymbol{c}_{\mathrm{host}}^{*}+m \boldsymbol{c}_{\mathrm{guest}}^{*}+n \boldsymbol{c}_{\mathrm{i}}^{*} .
$$

The indices $m$ and $n$ relate to $\gamma$ and $\delta$, respectively, with $\boldsymbol{c}_{\text {guest }}^{*}=\gamma \boldsymbol{c}_{\text {host }}^{*}$ and $\boldsymbol{c}_{\mathrm{i}}^{*}=\delta \boldsymbol{c}_{\text {host }}^{*}$.

Similar results were later reported [39] for $n$ hexadecane/urea, with phase I assigned to the $(3+1)$ dimensional superspace group $P 6_{1} 22(00 \gamma)$. However, in contrast to $n$-nonadecane/urea, phase II was reported to exhibit main reflections (i.e., $(h, k, l, m)$ with $l=0$ and/or $m=0$ ) with $h+k$ odd, but not to exhibit satellite reflections (i.e., $(h, k, l, m)$ with $l \neq 0$ and $m \neq 0)$ with $h+k$ odd, and phase II was described by the $(3+1)$-dimensional superspace group $P 2_{1} 2_{1} 2_{1}(00 \gamma)$ with $\gamma=0.486$. In phase III, many satellite reflections were observed with $h+k$ odd and were indexed in the $(3+2)$-dimensional superspace group $P 2_{1} 2_{1} 2_{1}(00 \gamma)(00 \delta)$, involving the additional misfit parameter $\delta=0.058$ coexisting with the misfit parameter $\gamma$ along the $\boldsymbol{c}$-axis [39].

In the present paper, we develop alternative interpretations of the superspace group descriptions and phase transitions in $n$-nonadecane/urea and $n$-hexadecane/urea, based in part on a high-resolution synchrotron singlecrystal X-ray diffraction study of $n$-nonadecane/urea.
Significantly, we demonstrate that $(3+1)$-dimensional superspace groups are sufficient to provide a complete description of the symmetry properties of the lowtemperature phases, in contrast to the un-necessarily elaborate $(3+2)$-dimensional superspace descriptions proposed previously $[11,36-42]$.

First, we consider the assertion $[36,38,42]$ that a $(3+2)$ dimensional superspace group representation is required to describe the symmetry properties of the low-temperature phases of $n$-nonadecane/urea (phases II and III) and $n$ hexadecane/urea (phase III). For $n$-nonadecane/urea, the temperature-independent misfit parameters were determined $[36,38,42]$ to be $\gamma=0.418$ and $\delta=0.090$ at ambient pressure. The X-ray diffraction data for $n$ nonadecane/urea recorded in the present work can also be indexed on the basis of the same $(3+2)$-dimensional description, with the same values of the misfit parameters $\gamma$ and $\delta$. However, on careful consideration of the indexing of reflections according to the $(3+2)$-dimensional superspace group representation, we have recognized that a simple relation actually exists between the values of the two misfit parameters $\gamma$ and $\delta$, specifically: $-2+5 \gamma=\delta$. It then follows that the reciprocal lattice basis vectors $\boldsymbol{c}_{\text {host }}^{*}$, $\boldsymbol{c}_{\text {guest }}^{*}$ and $\boldsymbol{c}_{\mathrm{i}}^{*}$ (see eq. (2)) are actually related by

$$
c_{\mathrm{i}}^{*}=-2 c_{\text {host }}^{*}+5 c_{\text {guest }}^{*} .
$$

On this basis, it is sufficient to describe the reciprocal space completely using a $(3+1)$-dimensional superspace description, with the following relationships between the indices in the $(3+2)$-dimensional $(h, k, l, m, n)$ and $(3+1)$ dimensional $\left(h, k, l^{\prime}, m^{\prime}\right)$ superspace descriptions:

$$
l^{\prime}=l-2 n \text { and } m^{\prime}=m+5 n .
$$

In the case of $n$-hexadecane/urea at ambient pressure, the superspace groups assigned previously [39] to phase II and phase III were $P 2_{1} 2_{1} 2_{1}(00 \gamma)$ and $P 2_{1} 2_{1} 2_{1}(00 \gamma)(00 \delta)$, respectively, and the temperature-independent misfit parameters were reported [39] to be $\gamma=0.486$ and $\delta=0.058$. Again, within experimental errors, we have identified a simple relation between $\gamma$ and $\delta$, in particular: $2-4 \gamma=\delta$. Within the $(3+2)$-dimensional superspace description for phase III, the reciprocal lattice basis vectors $\boldsymbol{c}_{\text {host }}^{*}, c_{\text {guest }}^{*}$ and $\boldsymbol{c}_{\mathrm{i}}^{*}$ (see eq. (2)) are actually related by

$$
c_{\mathrm{i}}^{*}=2 c_{\text {host }}^{*}-4 c_{\text {guest }}^{*} .
$$

Again, a (3+1)-dimensional superspace description is sufficient, with the following relationships between the indices in the $(3+2)$-dimensional $(h, k, l, m, n)$ and $(3+1)$ dimensional $\left(h, k, l^{\prime}, m^{\prime}\right)$ superspace descriptions:

$$
l^{\prime}=l+2 n \text { and } m^{\prime}=m-4 n .
$$

These simple relationships suggest that, for the lowtemperature phases of both $n$-hexadecane/urea and $n$ nonadecane/urea, the $(3+2)$-dimensional superspace group descriptions are not necessary. 


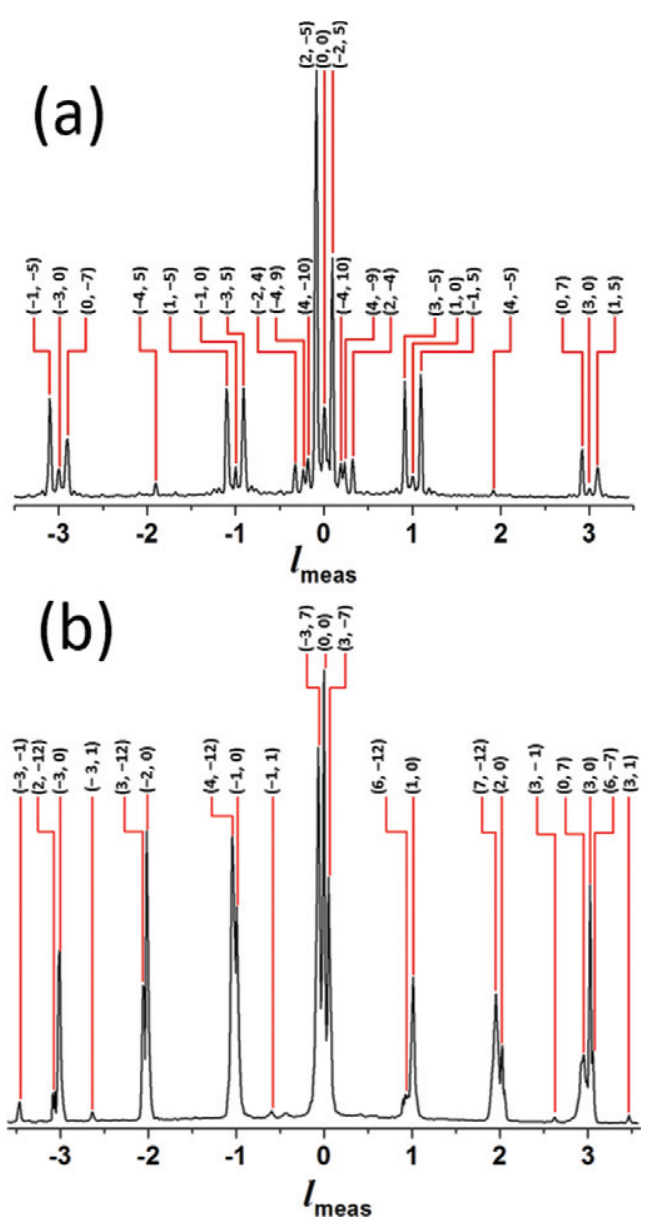

Fig. 2: (Colour online) One-dimensional $l$-scans through reciprocal space for $n$-nonadecane/urea at $147 \mathrm{~K}$ (phase II) with (a) $h=3, k=-2$, and (b) $h=3, k=1$. The position ( $\boldsymbol{q}$ ) along the $\boldsymbol{c}^{*}$-axis is given as $\boldsymbol{q}=l_{\text {meas }} \boldsymbol{c}_{\text {host }}^{*}$. The major observed reflections are labelled by the $\left(l^{\prime}, m^{\prime}\right)$ indices in the $(3+1)$-dimensional superspace description.

In our single-crystal X-ray diffraction study of $n$-nonadecane/urea, data were recorded for phase II at $147 \mathrm{~K}$ (i.e., just below the $\mathrm{I} \leftrightarrow \mathrm{II}$ transition temperature $T_{1}=157 \mathrm{~K}[59]$ ) and for phase III at $100 \mathrm{~K}$ (i.e., well below the II $\leftrightarrow$ III transition temperature $\left.T_{2}=140 \mathrm{~K}[59]\right)$. Representative one-dimensional $l$-scans through reciprocal space, parallel to the $\boldsymbol{c}_{\text {host }}^{*}$ and $\boldsymbol{c}_{\text {guest }}^{*}$ axes, are shown for phase II in fig. 2 and for phase III in fig. 3 (all $l$-scans were determined, using standard data analysis techniques, from experimental measurements of the complete reciprocal space; see the "Experimental details" section). For the $l$-scan shown in fig. $2(\mathrm{a})$, the indexing of the reflections based on both the $(3+2)$-dimensional $(h, k, l, m, n)$ and $(3+1)$-dimensional $\left(h, k, l^{\prime}, m^{\prime}\right)$ superspace descriptions is specified in table 1 , verifying that all reflections are correctly indexed in the $(3+1)$-dimensional superspace description.

For phase II, surprisingly, the most intense peaks in the scan with $h+k$ odd (fig. 2(a)) correspond to satellite reflections; significantly, however, weak main reflections of
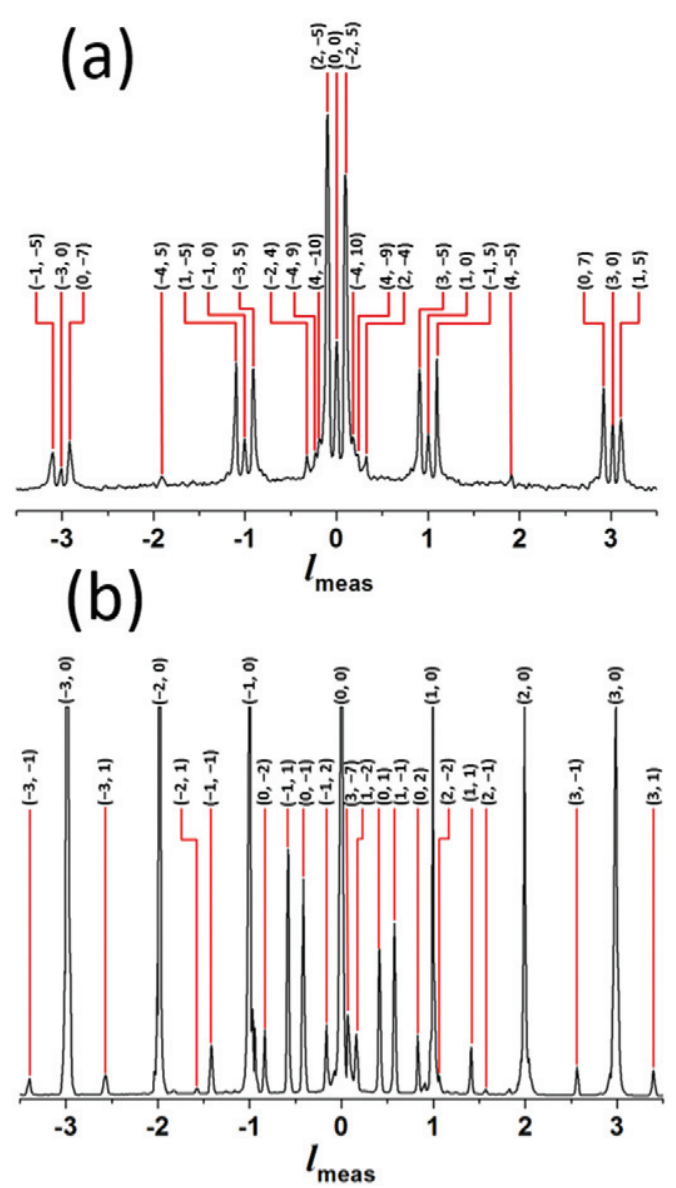

Fig. 3: (Colour online) One-dimensional $l$-scans through reciprocal space for $n$-nonadecane/urea at $100 \mathrm{~K}$ (phase III) with (a) $h=-3, k=-2$, and (b) $h=3, k=1$. The position ( $\boldsymbol{q})$ along the $\boldsymbol{c}^{*}$-axis is given as $\boldsymbol{q}=l_{\text {meas }} \boldsymbol{c}_{\text {host }}^{*}$. The major observed reflections are labelled by the $\left(l^{\prime}, m^{\prime}\right)$ indices in the $(3+1)$-dimensional superspace description.

both $\left(h, k, l^{\prime}, 0\right)$ and $\left(h, k, 0, m^{\prime}\right)$ types are also observed. In the scan with $h+k$ even (fig. $2(\mathrm{~b})$ ), main reflections of both $\left(h, k, l^{\prime}, 0\right)$ and $\left(h, k, 0, m^{\prime}\right)$ types are observed, as well as satellite reflections $\left(h, k, l^{\prime}, m^{\prime}\right)$ with $l^{\prime} \neq 0$ and $m^{\prime} \neq 0$.

For phase III, the corresponding scans with $h+k$ odd (fig. 3(a)) or $h+k$ even (fig. 3(b)) reveal a significant increase in the intensities of the main reflections of both $\left(h, k, l^{\prime}, 0\right)$ and $\left(h, k, 0, m^{\prime}\right)$ types, but there are no marked changes in the intensities of the satellite reflections.

The major difference between our data and those reported previously $[36,38,42]$ is that, in phase II, we observe main reflections of both $\left(h, k, l^{\prime}, 0\right)$ and $(h, k, 0$, $\left.m^{\prime}\right)$ types with $h+k$ odd, although, as noted above, we emphasize that the main reflections with $h+k$ odd generally have lower intensities than the satellite reflections with the same $h$ and $k$ values. Clearly, we conclude that, on passing from phase I to phase II, the C-centering of the orthohexagonal unit cell is lost for both the basic host structure and the basic guest structure. 
Referring momentarily to the $(3+2)$-dimensional superspace group description, we note that the reflection condition [60] $((h, k, l, m, n): h+k+n=2 N ; N=$ integer) for the superspace group $C 222_{1}(00 \gamma)(10 \delta)$ proposed previously $[38,42]$ for phase II of $n$-nonadecane/urea is not supported by our X-ray diffraction data. For example, the satellite reflections indexed in the $(3+2)$ dimensional system as $(3,-2,0,0,2)$ and $(3,-2,0,1,-2)$, which should be absent for superspace group $C 222_{1}(00 \gamma)$ $(10 \delta)$, are clearly observed in our X-ray diffraction data. These reflections correspond (using the relations in eq. (4)) to $(3,-2,-4,10)$ and $(3,-2,4,-9)$, respectively, in the $(3+1)$-dimensional description used to index the reflections in fig. 2(a).

In the frame of the $(3+2)$-dimensional superspace proposed previously for $n$-nonadecane/urea $[38,42]$, the observed reflections in phase II are actually compatible with superspace group $P 2_{1} 2_{1} 2_{1}(00 \gamma)(00 \delta)$ [60], rather than superspace group $C 222_{1}(00 \gamma)(10 \delta)$. We note that $P 2_{1} 2_{1} 2_{1}(00 \gamma)(00 \delta)$ is the same superspace group as phase III, and is a subgroup of $C 222_{1}(00 \gamma)(10 \delta)$ [29].

However, as we have already shown that a $(3+1)$ dimensional superspace is sufficient to describe the symmetry properties of these incommensurate materials, we propose that, for both $n$-nonadecane/urea and $n$ hexadecane/urea, phase II and phase III are both fully described by the $(3+1)$-dimensional superspace group $P 2_{1} 2_{1} 2_{1}(00 \gamma)$. For each material, the primary difference between phase II and phase III is that the intensities of the main reflections $\left(\left(h, k, l^{\prime}, 0\right)\right.$ and $\left.\left(h, k, 0, m^{\prime}\right)\right)$ and the satellite reflections $\left(\left(h, k, l^{\prime}, m^{\prime}\right)\right.$ with $l^{\prime} \neq 0$ and $\left.m^{\prime} \neq 0\right)$ have significantly different temperature dependences when $h+k$ is odd.

We emphasize that our conclusions are fully corroborated by the analysis of several other one-dimensional $l$-scans for phases II and III of $n$-nonadecane/urea, in addition to those shown in figs. 2 and 3 . We also note that most reflections in table 1 have large values of the indices $\left(l^{\prime}, m^{\prime}\right)$ in the $(3+1)$-dimensional superspace description, whereas the values of the indices $(l, m, n)$ in the $(3+2)$ dimensional superspace description are smaller. Examples of incommensurate systems with high indices for satellite reflections arise when the modulation function becomes discontinuous and a non-analytic "soliton regime" can exist, allowing higher harmonics to be observed $[61,62]$. Importantly, in the present case, the modulation function is discontinuous by construction, as it is interrupted by the specific periodic $\mathrm{CH}_{3} \cdots \mathrm{H}_{3} \mathrm{C}$ interactions that exist between the ends of adjacent $n$-alkane guests.

In contrast to our $(3+1)$-dimensional superspace, we emphasize that the $(3+2)$-dimensional superspace gives a huge number of possible solutions for indexing each experimentally observed reflection. For example, the reflection observed in fig. 2(a) (see table 1) at $l_{\text {meas }}=0.914$ is indexed unambiguously and uniquely in our $(3+1)$ dimensional superspace as $(3,-2,3,-5)$, with $l_{\text {calc }}=$ 0.910 , but can be indexed in the $(3+2)$-dimensional
Table 1: Comparison of the measured $l_{\text {meas }}$ (extracted from the data in fig. 2(a)) and calculated $l_{\text {calc }}$ positions along the $\boldsymbol{c}^{*}$-axis for all significant reflections in the one-dimensional reciprocalspace scan at $h=3$ and $k=-2$ for $n$-nonadecane/urea at $147 \mathrm{~K}$ (phase II). The position $(\boldsymbol{q})$ along the $\boldsymbol{c}^{*}$-axis is given as $\boldsymbol{q}=l_{\text {meas }} \boldsymbol{c}_{\text {host }}^{*}$ or $\boldsymbol{q}=l_{\text {calc }} \boldsymbol{c}_{\text {host }}^{*}$. The indexing $\left(3,-2, l^{\prime}, m^{\prime}\right)$ in the $(3+1)$-dimensional superspace and $(3,-2, l, m, n)$ in the $(3+2)$-dimensional superspace are specified. The calculated values $l_{\text {calc }}$ are determined from the set of indices $\left(l^{\prime}, m^{\prime}\right)$ or $(l, m, n)$ using $l_{\text {calc }}=l^{\prime}+\gamma m^{\prime}=l+\gamma m+\delta n$. For the $(3+2)$ dimensional superspace, the assignment of indices $(l, m, n)$ is not unique (see text), and only the set of indices of lowest value is reported here.

\begin{tabular}{cccl}
\hline$l_{\text {meas }}$ & $l_{\text {calc }}$ & $\left(l^{\prime}, m^{\prime}\right)$ & $(l, m, n)$ \\
\hline 0.095 & 0.090 & $(-2,5)$ & $(0,0,1)$ \\
0.189 & 0.180 & $(-4,10)$ & $(0,0,2)$ \\
0.232 & 0.238 & $(4,-9)$ & $(0,1,-2)$ \\
0.327 & 0.328 & $(2,-4)$ & $(0,1,-1)$ \\
0.914 & 0.910 & $(3,-5)$ & $(1,0,-1)$ \\
0.999 & 1.000 & $(1,0)$ & $(1,0,0)$ \\
1.093 & 1.090 & $(-1,5)$ & $(1,0,1)$ \\
1.913 & 1.910 & $(4,-5)$ & $(2,0,-1)$ \\
2.922 & 2.926 & $(0,7)$ & $(2,2,1)$ \\
2.996 & 3.000 & $(3,0)$ & $(3,0,0)$ \\
3.090 & 3.090 & $(1,5)$ & $(3,0,1)$ \\
\hline
\end{tabular}

superspace by any of the reflections $(3,-2,1,0,-1)$, $(3,-2,3,-5,0),(3,-2,-1,5,-2)$ or $(3,-2,5,-10,1)$, etc., all of which give the same value of $l_{\text {calc }}=0.910$. Clearly, such ambiguity and non-uniqueness in the indexing of reflections is another unsatisfactory feature of the $(3+2)$-dimensional superspace description.

In conclusion, we have shown that rationalization of the diffraction patterns of $n$-nonadecane/urea and $n$-hexadecane/urea inclusion compounds in their lowtemperature phases does not require the introduction of a fifth dimension in superspace, as proposed previously, and does not require new concepts, such as "hidden degrees of freedom in superspace" [36], to be invented. As a consequence, the temperature-independent misfit parameter $\gamma$ is the only aperiodicity in these materials, and, furthermore, the misfit parameter $\gamma$ is a common feature in all phases. The most probable mechanism responsible for the phase transitions is then related to "classical" degrees of freedom of the rigid $n$-alkane guest molecules inside the urea tunnels (i.e., reorientational motions of the guest molecules about the host tunnel axis (orientational ordering) and translational motions of the guest molecules along this axis), resulting in a model of pseudo-spin translation-rotation coupling which was already suggested several years ago [58]. Indeed, simple group theoretical considerations show that, in the present case, two different order parameters are necessary. One order parameter belongs to the $E_{2}$ irreducible representation at the zone centre of the Brillouin zone ( $\Gamma$-point), 
responsible for the strong orthorhombic distortion, and the other order parameter belongs to the $M_{2}$ representation at the Brillouin zone boundary $M$-point, responsible for the loss of C-centering in the orthorhombic phases [63]. In the frame of a translation-rotation coupling model, convenient pseudo-spin coordinates at the $\Gamma$ and/or $M$ points describing reorientational ordering coupled to the translational motions of the guest molecules at the $M$-point must be considered as order parameters. Importantly, different couplings between these variables may explain the differences observed in the phase transition sequences between $n$-nonadecane/urea and $n$-hexadecane/urea. The development of this model will be presented in a forthcoming paper.

Experimental details. - Crystals of the $n$ nonadecane/urea inclusion compound were prepared by the following method. An excess of $n$-nonadecane was added to a saturated solution of urea in methanol in a conical flask at $55^{\circ} \mathrm{C}$. 2-Methyl-2-butanol was then added dropwise until the solution was homogeneous. Any precipitate formed at this stage was dissolved by further addition of the minimum amount of methanol required for complete dissolution. The flask was stoppered and placed inside an incubator, which was cooled slowly from $55^{\circ} \mathrm{C}$ to $0{ }^{\circ} \mathrm{C}$. The crystals formed were collected by filtration and washed with 2, 2, 4-trimethylpentane (to remove any $n$-nonadecane adhering to their external surfaces).

Single-crystal X-ray diffraction data were recorded for $n$-nonadecane/urea on beamline I19 (EH1) at Diamond Light Source, with temperature controlled by an Oxford Cryosystems Cryostream Plus open-flow nitrogen gas cryostat. The data were measured using a Rigaku Crystal Logic Kappa goniometer with a Saturn $724+$ detector at the zirconium absorption edge $(\lambda=0.6889 \AA)$. At $180 \mathrm{~K}$, $147 \mathrm{~K}$ and $100 \mathrm{~K}$, a full sphere of data to a resolution of $0.8 \AA$ was collected with a scan width of $0.3^{\circ}$. At ambient temperature, only indexing frames were collected. Raw frame data were processed using CrysAlisPro from Agilent Technologies (now Rigaku Oxford Diffraction). For the data recorded at $147 \mathrm{~K}$ (phase II) and $100 \mathrm{~K}$ (phase III), after peak hunting and indexing, a unit cell with orthorhombic metric symmetry $(a \approx 14.1 \AA, b \approx 8.15 \AA$, $c \approx 10.99 \AA$ ) was used (with some variation in unit cell parameters at the different temperatures). From the collected data, reconstructed precession images were generated for the regions of reciprocal space of interest. To generate one-dimensional $l$-scans through reciprocal space along the $\boldsymbol{c}^{*}$-axis (as shown in figs. 2 and 3), an appropriate $h k l$ layer in reciprocal space (with one of the values of $h$ or $k$ fixed) was reconstructed to a resolution of $2 \AA$ or $3 \AA$. From the reconstructed layer, a line drag was performed (using CrysAlisPro) at fixed $h$ and $k$, moving from positive to negative values of $l$, giving a continuous plot of intensity vs. $l$. Within these reconstructed $l$-scans, the spacing between adjacent digitized data points corresponds to $\Delta l \approx 0.01$ for fig. 2 (a) and $\Delta l \approx 0.007$ for fig. 2(b), 3(a) and 3(b); thus, the experimental error in assessing the positions of peak maxima in these $l$-scans is of the order of $\delta l= \pm 0.005$ and $\delta l= \pm 0.0035$, respectively.

$$
* * *
$$

We are grateful to EPSRC (studentship to BAP) and Cardiff University for financial support. We acknowledge Diamond Light Source for the award of experimental time on beamline I16 (proposal MT6361) and on beamline I19 (proposal MT6365). We thank Dr Benson Kariuki for helpful discussions and Dr Colan Hughes for preparing fig. 1(a).

\section{REFERENCES}

[1] BAK P., Rep. Prog. Phys., 45 (1982) 587.

[2] Shechtman D., Blech I., Gratias D. and Cahn J. W., Phys. Rev. Lett., 53 (1984) 1951.

[3] Dubois J. M., Useful Quasicrystals (World Scientific, Singapore) 2005.

[4] Steurer W. and Sutter-Widmer D., J. Phys. Appl. Phys., 40 (2007) R229.

[5] Smith A. E., Acta Crystallogr., 5 (1952) 224.

[6] Harris K. D. M. and Thomas J. M., J. Chem. Soc. Faraday Trans., 86 (1990) 2985.

[7] Harris K. D. M., J. Solid State Chem., 106 (1993) 83.

[8] Hollingsworth M. D. and Harris K. D. M., in Comprehensive Supramolecular Chemistry, edited by ATwooD A. L., Davies J. E. D., MacNicol D. D. and Vogtle F., Vol. 6 (Pergamon Press, Oxford) 1996, pp. 177-237.

[9] Guillaume F., J. Chim. Phys. Phys.-Chim. Biol., 96 (1999) 1295.

[10] Harris K. D. M., Supramol. Chem., 19 (2007) 47.

[11] Mariette C., Guérin L., Rabiller P., Ecolivet C., García-Orduña P., Bourges P., Bosak A., de Sanctis D., Hollingsworth M. D., Janssen T. and Toudic B., Phys. Rev. B, 87 (2013) 104101.

[12] Hollingsworth M. D., Brown M. E., Hillier A. C., Santarsiero B. D. and Chaney J. D., Science, 273 (1996) 1355.

[13] Lee S. O. and Harris K. D. M., Chem. Phys. Lett., 307 (1999) 327.

[14] Kelly N. E., Lee S. O. and Harris K. D. M., J. Am. Chem. Soc., 123 (2001) 12682.

[15] Palmer B. A., Harris K. D. M. and Gulllaume F., Angew. Chem., Int. Ed., 49 (2010) 5096.

[16] Palmer B. A., Le Comte A., Harris K. D. M. and Guillaume F., J. Am. Chem. Soc., 135 (2013) 14512.

[17] Collins S. P., Laundy D., Harris K. D. M., Kariuki B. M., Bauer C. L., Brown S. D. and Thompson P., J. Phys.: Condens. Matter, 14 (2002) 123.

[18] Palmer B. A., Edwards-Gau G. R., Kariuki B. M., Harris K. D. M., Dolbnya I. P., Collins S. P. and Sutter J. P., J. Phys. Chem. Lett., 6 (2015) 561.

[19] Marti-Rujas J., Desmedt A., Harris K. D. M. and Guillaume F., J. Am. Chem. Soc., 126 (2004) 11124.

[20] Martí-Rujas J., Desmedt A., Harris K. D. M. and Guillaume F., J. Phys. Chem. B, 111 (2007) 12339.

[21] Martí-Rujas J., Harris K. D. M., Desmedt A. and Guillaume F., J. Phys. Chem. B, 110 (2006) 10708. 
[22] Martí-Rujas J., Desmedt A., Harris K. D. M. and Guillaume F., J. Phys. Chem. C, 113 (2009) 736.

[23] Rennie A. J. O. and Harris K. D. M., Proc. R. Soc. A, 430 (1990) 615.

[24] Harris K. D. M. and Hollingsworth M. D., Proc. R. Soc. A, 431 (1990) 245.

[25] Schmicker D., van Smaalen S., de Boer J. L., Haas C. and Harris K. D. M., Phys. Rev. Lett., 74 (1995) 734.

[26] George A. R. and Harris K. D. M., J. Mol. Graph., 13 (1995) 138.

[27] Lefort R., Etrillard J., Toudic B., Guillaume F., Breczewsiki T. and Bourges P., Phys. Rev. Lett., 77 (1996) 4027.

[28] Weber T., Boysen H., Honal M., Frey F. and Neder R. B., Z. Kristallogr., 211 (1996) 238.

[29] van Smaalen S. and Harris K. D. M., Proc. R. Soc. A, 452 (1996) 677.

[30] Ollivier J., Ecolivet C., Beaufils S., Guillaume F. and Breczewski T., Europhys. Lett., 43 (1998) 546.

[31] Etrillard J., Lasjaunias J. C., Toudic B., Guillaume F. and Breczewski T., Europhys. Lett., 49 (2000) 610.

[32] Le Lann H., Odin C., Toudic B., Ameline J. C., Gallier J., Guillaume F. and Breczewsik T., Phys. Rev. B, 62 (2000) 5442.

[33] Lefort R., Toudic B., Etrillard J., Guillaume F., Bourges P., Currat R. and Breczewski T., Eur. Phys. J. B, 24 (2001) 51.

[34] Toudic B., Aubert F., Ecolivet C., Bourges P. and Breczewski T., Phys. Rev. Lett., 96 (2006) 145503.

[35] Harris K. D. M., in Turning Points in SolidState, Materials and Surface Science, edited by HARRIS K. D. M. and Edwards P. P. (Royal Society of Chemistry, Cambridge) 2008, pp. 302-33.

[36] Toudic B., Garcia P., Odin C., Rabiller P., Ecolivet C., Collet E., Bourges P., McIntyre G. J., Hollingsworth M. D. and Breczewski T., Science, 319 (2008) 69.

[37] Toudic B., Lefort R., Ecolivet C., Guérin L., Currat R., Bourges P. and Breczewski T., Phys. Rev. Lett., 107 (2011) 205502.

[38] Toudic B., Rabiller P., Bourgeois L., Huard M., Ecolivet C., McIntyre G. J., Bourges P., Breczewski T. and Janssen T., EPL, 93 (2011) 16003.

[39] Huard M., Toudic B., Rabiller P., Ecolivet C., Guérin L., Bourges P., Breczewsiki T. and Hollingsworth M. D., J. Chem. Phys., 135 (2011) 204505.

[40] Mariette C., Huard M., Rabiller P., Nichols S. M., Ecolivet C., Janssen T., Alquist K. E. III, Hollingsworth M. D. and Toudic B., J. Chem. Phys., 136 (2012) 104507.
[41] Mariette C., Guérin L., Rabiller P., Chen Y. S., Bosak A., Popov A., Hollingsworth M. D. and Toudic B., Z. Kristallogr., 230 (2014) 5.

[42] Zerdane S., Mariette C., Mcintyre G. J., LeméeCailleau M. H., Rabiller P., Guérin L., Ameline J. C. and Toudic B., Acta Crystallogr. Sect. B, 71 (2015) 293.

[43] Guérin L., Mariette C., Rabiller P., Huard M., Ravy S., Fertey P., Nichols S. M., Wang B., Mannsfeld S. C. B., Weber T., Hollingsworth M. D. and Toudic B., Phys. Rev. B, 91 (2015) 184101.

[44] Harris K. D. M. and Jonsen P., Chem. Phys. Lett., 154 (1989) 593.

[45] Guillaume F., Sourisseau C. and Dianoux A., J. Chem. Phys., 93 (1990) 3536.

[46] Guillaume F., Sourisseau C. and Dianoux A., J. Chim. Phys. Phys.-Chim. Biol., 88 (1991) 1721.

[47] Souaille M., Guillaume F. and Smith J. C., J. Chem. Phys., 105 (1996) 1516.

[48] Souaille M., Guillaume F. and Smith J. C., J. Chem. Phys., 105 (1996) 1529.

[49] Sounille M., Smith J. C. and Gulllaume F., J. Phys. Chem. B, 101 (1997) 6753.

[50] Harris K. D. M., J. Mol. Struct., 374 (1996) 241.

[51] Pemberton R. C. and Parsonage N. G., Trans. Faraday Soc., 61 (1965) 2112.

[52] Pemberton R. C. and Parsonage N. G., Trans. Faraday Soc., 62 (1966) 553.

[53] Forst R., Jagodzinski H., Boysen H. and Frey F., Acta Crystallogr. Sect. B, 43 (1987) 187.

[54] Forst R., Jagodzinski H., Boysen H. and Frey F., Acta Crystallogr. Sect. B, 46 (1990) 70.

[55] Fukao K., Horiuchi T., Taki S. and Matsushige K., Mol. Cryst. Liq. Cryst., 180 (1990) 405.

[56] Fukao K., J. Chem. Phys., 92 (1990) 6867.

[57] Harris K. D. M., Gameson I. and Thomas J. M., J. Chem. Soc. Faraday Trans., 86 (1990) 3135.

[58] Lynden-Bell R. M., Mol. Phys., 79 (1993) 313.

[59] López-Echarri A., Ruiz-Larrea I., FraileRodríguez A., Díaz-Hernández J., Breczewski T. and Bocanegra E. H., J. Phys.: Condens. Matter, 19 (2007) 186221.

[60] Stokes H. T., Campbell B. J. and van Smaalen S., Acta Crystallogr. Sect. A, 67 (2011) 45; http://stokes. byu.edu/ssg.html.

[61] Pérez-Mato J. M. and Madariaga G., Solid State Commun., 58 (1986) 105.

[62] Marmeggi J. C., Lander G. H., van Smaalen S., BrüChel T. and Zeyen C. M. E., Phys. Rev. B, 42 (1990) 9365.

[63] Stokes H. T. and Hatch D. M., Isotropy Subgroups of the 230 Crystallographic Space Groups (World Scientific, Singapore) 1988. 